\title{
A GENERALIZATION OF THE POISSON TRANSFORM ${ }^{1}$
}

\section{CHARLES STANDISH}

In this paper we shall obtain inversion formulas in terms of differential operators for the transforms

$$
f(z)=\frac{1}{\pi} \int \frac{A(z-t)+B}{(z-t)^{2}+C^{2}} d \alpha(t)
$$

where $\alpha(t)$ is of bounded variation in every finite interval, and

$$
f(z)=\frac{1}{\pi} \int \frac{A(z-t)+B}{(z-t)^{2}+C^{2}} \phi(t) d t
$$

where $\phi(t)$ is Lebesgue integrable in every finite interval. In both cases the integral is to be interpreted as

$$
\lim _{R \rightarrow \infty, S \rightarrow \infty} \int_{-S}^{R}
$$

When limits are omitted from an integral appearing in the text the range of integration is understood to be $(-\infty, \infty)$.

At the conclusion of the paper we shall give a complex inversion formula for (2).

The preceding transforms include as special cases the Poisson transform $(A=0, B=C=1)$ recently studied by Pollard [1], and the "conjugate" of the Poisson transform $(B=0, A=C=1)$ whose kernel is the Hilbert transform of the Poisson kernel.

The inversion of (1) and (2) is intended as a first step toward the solution of the problem of inverting the convolution transform whose kernel is a rational function with no poles on the real axis. I am indebted to Professor Pollard for proposing the problem considered in this paper.

For the heuristic motivation of our inversion formulas we refer to [1] and [2]. We distinguish two cases according as $A$ is or is not zero. In the former case a slight modification of Pollard's proof for the Poisson case shows that (1) is inverted by

Presented to the Society, December 27, 1954 under the title Inversion of a generalized Poisson transform; received by the editors September 27, 1954, and, in revised form, February 11, 1955.

1 Research sponsored by the Office of Ordnance Research, U. S. Army, under Contract No. DA-30-115-ORD-439. 


$$
\begin{aligned}
\lim _{t \rightarrow 1} \int_{0}^{x} G e^{|t C H|} B|C|^{-1} f(u) d u \\
\quad=\frac{1}{2}[\alpha(x+0)+\alpha(x-0)]-\frac{1}{2}[\alpha(0+)+\alpha(0-)]
\end{aligned}
$$

for every $x$ and (2) by

$$
\lim _{t \rightarrow 1} G e^{|t C H|} B|C|^{-1} f(x)=\phi(x)
$$

for almost all $x$, where

$$
\begin{aligned}
& G=C^{2}\left(B^{2}+C^{2} A^{2}\right)^{-1}, \quad H=(1 / i) D, \\
& e^{|t C H|} f(x)=\cos C t D f(x)+D^{-1} \sin C t D \widehat{f}(x), \\
& \cos C t D f(x)=\sum_{K=0}^{\infty} \frac{(-1)^{K}(C t)^{2 K}}{(2 K) !} f(x)^{(2 K)}, \\
& D^{-1} \sin C t D \hat{f}(x)=\sum_{K=0}^{\infty} \frac{(-1)^{K}(C t)^{2 K+1}}{(2 K+1) !} \widehat{f}(x)^{(2 K),} \\
& \hat{f}(x)=-\pi^{-1} \int_{0}^{\infty} u^{-2}[f(x+u)+f(x-u)-2 f(x)] d u .
\end{aligned}
$$

In the case $A \neq 0$ we shall show that according as $f(x)$ is in the form (1) or (2)

$$
\begin{aligned}
\lim _{t \rightarrow 1} & \int_{\theta}^{x} G e^{|t C H|} h(u) d u \\
& =\frac{1}{2}[\alpha(x+0)+\alpha(x-0)-(\alpha(0+)+\alpha(0-))]-A G K x
\end{aligned}
$$

for every $x$,

$$
\lim _{t \rightarrow 1} G e^{|t C H|} h(x)=\phi(x)-A G K
$$

for almost all $x$, where

$$
\begin{aligned}
h(x) & =-A \int_{0}^{x} \hat{f}(u) d u+B|C|^{-1} f(x), \\
K & =-2\left(A^{2} G \log 4\right)^{-:} \lim _{\lambda \rightarrow \infty} \int_{\lambda}^{2 \lambda} \frac{-A t+B}{C^{2}+t^{2}} d \alpha^{\sharp}(t)
\end{aligned}
$$

$\alpha^{f}(x)$ denoting the left-hand side of (III).

To obtain a complex inversion formula for (2) we proceed formally as follows. Define 


$$
\begin{aligned}
\cos C t D g(x)= & 2^{-1}\left[e^{i C t D} g(x)+e^{-i C t D} g(x)\right] \\
= & 2^{-1}[g(x+i C t)+g(x-i C t)], \\
\sin C t D g(x)= & (2 i)^{-1}\left[e^{i C t D} g(x)-e^{-i C t D} g(x)\right] \\
= & (2 i)^{-1}[g(x+i C t)-g(x-i C t)], \\
& h^{b}(x)=\int_{0}^{x} \hat{h}(u) d u,
\end{aligned}
$$

then (IV) becomes formally

$$
\begin{aligned}
\lim _{t \rightarrow 1} G e^{|t C H|} h(x)= & \lim _{t \rightarrow 1} 2^{-1}[h(x+i C t)+h(x-i C t)] \\
& +\lim _{t \rightarrow 1} 2^{-1}\left[h^{b}(x+i C t)+h^{b}(x-i C t)\right] \\
= & \phi(x)-A K .
\end{aligned}
$$

These conclusions will be obtained rigorously in the final section of this paper.

1. Properties of the transform. We summarize in Theorems 1.1 and 1.2 the properties of (1) and (2) needed in the sequel. Proofs are omitted since they are similar to the proofs for the Poisson case to be found in [1].

THEOREM 1.1. If the transform

$$
f(z)=\pi^{-1} \int \frac{A(z-t)+B}{(z-t)^{2}+C^{2}} d \alpha(t), \quad z=x+i y,
$$

converges for a single value of $z$ in the strip $|y|<|C|$ it converges uniformly in any compact subset of this strip and defines a function analytic there. Thus we may differentiate an arbitrary number of times under the integral sign.

TheOREM 1.2. If (1.1) converges at a point $z_{0}$ in $|y|<|C|$ then

$$
\begin{array}{ll}
\alpha(t)=o\left(t^{2}\right), & A=0, \\
\alpha(t)=o(t), & A \neq 0,
\end{array}
$$

2. The function $\hat{f}(x)$. We shall demonstrate here the existence of $\hat{f}(x)$ for all $f(x)$ defined by (1.1).

THEOREM 2.1. For all $f(x)$ representable in the form (1.1)

$$
\widehat{f}(x)=-\pi^{-1} \int_{0}^{\infty} u^{-2}[f(x+u)+f(x-u)-2 f(x)] d u
$$


exists as an analytic function for all real $x$ and

$$
\text { (2.1) } f(x)=-\pi^{-1} \int \frac{B(x-t)^{2}-2 A C^{2}(x-t)-B C^{2}}{C\left[(x-t)^{2}+C^{2}\right]^{2}} d \alpha(t) \text {. }
$$

The proof follows along lines similar to the argument for the Poisson case but the details are sufficiently complicated to warrant carrying out the demonstration.

By (1.1)

$$
\begin{aligned}
u^{-2}[f(x+ & u)+f(x-u)-2 f(x)] \\
& =\pi^{-1} \int u^{-2}[\lambda(x+u-t)+\lambda(x-u-t)-2 \lambda(x-t)] d \alpha(t)
\end{aligned}
$$

where

$$
\lambda(x)=(A x+B) /\left(x^{2}+C^{2}\right),
$$

the integral converging uniformly for fixed $x$ in any interval $-R \leqq u$ $\leqq R$ as can be shown by the argument used to establish Theorem 1.1. Integrating under the integral sign from 0 to $R$ and letting $x-t=V$ we obtain

$$
\begin{aligned}
- & \pi^{-1} \int_{0}^{R} u^{-2}[f(x+u)-2 f(x)+f(x-u)] d u \\
= & -\pi^{-2} \int \frac{B V^{2}-2 A C^{2} V-B C^{2}}{C\left(V^{2}+C^{2}\right)^{2}}\left[\arctan \left(\frac{R+V}{C}\right)\right. \\
& \left.+\arctan \left(\frac{R-V}{C}\right)\right] d V \alpha(x-V) \\
& -\left(2 \pi^{2}\right)^{-1} \int \frac{A V^{2}+2 B V-A C^{2}}{\left(V^{2}+C^{2}\right)^{2}} \log \frac{(V+R)^{2}+C^{2}}{(V-R)^{2}+C^{2}} d V \alpha(x-V) \\
= & I_{1}+I_{2} .
\end{aligned}
$$

Defining

$$
G(V)=\pi^{-1} \int_{-\infty}^{V} \frac{B \xi^{2}-2 A C^{2} \xi-B C^{2}}{C\left(\xi^{2}+C^{2}\right)^{2}} d \alpha_{\xi}(x-\xi)
$$

(the existence of $G(V)$ follows readily from an integration by parts upon noting the order properties of $\alpha(t)$ obtained from Theorem 1.2. Indeed, $G(\infty)$ is finite, a fact we shall need subsequently), we have

$$
I_{1}=-\pi^{-1} \int\left[\arctan \frac{(R+V)}{C}+\arctan \frac{(R-V)}{C}\right] d G(V) .
$$


Integrating by parts we obtain

$$
\begin{aligned}
I_{1} & =-\pi^{-1} \int C G(V)\left[\left(C^{2}+(R+V)^{2}\right)^{-1}-\left(C^{2}+(R-V)^{2}\right)^{-1}\right] d V \\
& =\pi^{-1} \int C[G(V-R)-G(V+R)]\left(C^{2}+V^{2}\right)^{-1} d V
\end{aligned}
$$

and by dominated convergence we have, upon letting $R$ tend to $\infty$, $\lim _{R \rightarrow \infty} I_{1}=-G(\infty)=-\pi^{-1} \int \frac{B(x-t)^{2}-2 A C^{2}(x-t)-B C^{2}}{C\left[(x-t)^{2}+C^{2}\right]^{2}} d \alpha(t)$.

We now show that $\lim _{R \rightarrow \infty} I_{2}=0$,

$$
\begin{aligned}
I_{2}= & -\left(2 \pi^{2}\right)^{-1} \int \frac{A V^{2}+2 B V-A C^{2}}{\left(V^{2}+C^{2}\right)^{2}} \log \frac{(V+R)^{2}+C^{2}}{(V-R)^{2}+C^{2}} d V \alpha(x-V) \\
= & -A\left(2 \pi^{2}\right)^{-1} \int \frac{V^{2}}{\left(V^{2}+C^{2}\right)^{2}} \log \frac{(V+R)^{2}+C^{2}}{(V-R)^{2}+C^{2}} d V \alpha(x-V) \\
& +\left(2 \pi^{2}\right)^{-1} \int \frac{A C^{2}-2 B V}{\left(V^{2}+C^{2}\right)^{2}} \log \frac{(V+R)^{2}+C^{2}}{(V-R)^{2}+C^{2}} d V \alpha(x-V) \\
= & I_{2}^{\prime}+I_{2}^{\prime \prime} .
\end{aligned}
$$

Consider $I_{2}^{\prime}$ : Let

$$
H(V)=-A\left(2 \pi^{2}\right)^{-1} \int_{-\infty}^{V} \frac{\xi}{C^{2}+\xi^{2}} d_{\xi} \alpha(x-\xi)
$$

then

$$
I_{2}^{\prime}=\int \frac{V}{\left(V^{2}+C^{2}\right)} \log \frac{(V+R)^{2}+C^{2}}{(V-R)^{2}+C^{2}} d H(V)
$$

and upon integrating by parts it can be shown that $I_{2}^{\prime}$ approaches zero as $R \rightarrow \infty$. For details we refer to $[1$, p. 545$]$. A similar treatment establishes that $I_{2}^{\prime \prime}$ approaches zero as $R \rightarrow \infty$. Our formula for $\widehat{f}(z)$ having been established it may be shown by the methods employed in the proof of Theorem 1.1 that $\hat{f}(z)$ is an analytic function for $|y|<|C|$. This completes the proof of Theorem 2.1.

3. Real inversion formulas. We give a series of lemmas which culminate in a proof of the inversion formulas (III) and (IV).

Lemma 3.1. With $\widehat{f}(x)$ defined as in Theorem $2.1, \int_{0}^{x} \widehat{f}(u) d u$ exists and 


$$
\begin{aligned}
\int_{0}^{x} \widehat{f}(u) d u= & -A C \pi^{-1} \int \frac{d \alpha(y)}{C^{2}+(x-y)^{2}} \\
& +B(\pi|C|)^{-1} \int \frac{(x-y) d \alpha(y)}{C^{2}+(x-y)^{2}}+K
\end{aligned}
$$

where

$$
K=A|C| \pi^{-1} \int \frac{d \alpha(y)}{C^{2}+y^{2}}+B(\pi|C|)^{-1} \int \frac{y d \alpha(y)}{C^{2}+y^{2}} .
$$

The integral obviously exists since $\widehat{f}(x)$ is analytic on the whole real line. To see that it is of the form (3.1) we write (2.1) in the form

$$
\begin{aligned}
\widehat{f}(x)= & -A i(2 \pi)^{-1} \int d \alpha(y) \int e^{i u(x-y)} u e^{-|C u|} d u \\
& +B(2 \pi C)^{-1} \int d \alpha(y) \int e^{i u(x-y)} u \operatorname{sgn} u e^{-|C u|} d u .
\end{aligned}
$$

Integrating both sides and noting that the uniform convergence of (3.2) in $x$ enables us to carry the integration under the inner integral we have our result. As an immediate consequence of the above we have

LEMMA 3.2.

$$
\begin{aligned}
B|C|^{-1} f(x)-A \int_{0}^{x} \hat{f}(u) d u & \\
& =(\pi|C|)^{-1}\left(B^{2}+A^{2} C^{2}\right) \int \frac{d \alpha(y)}{C^{2}+(x-y)^{2}}-A K .
\end{aligned}
$$

A slight modification of the proof of the inversion formula for the Poisson transform given in [1] yields the proof of

LEMMA 3.3. The transforms

$$
f_{1}(x)=\pi^{-1} \int \frac{d \alpha(y)}{C^{2}+(x-y)^{2}}, \quad f_{2}(x)=\pi^{-1} \int \frac{\phi(y) d y}{C^{2}+(x-y)^{2}}
$$

are inverted by

$$
\begin{aligned}
C \lim _{t \rightarrow 1} \int_{0}^{x} e^{|\iota C H|} f_{1}(u) d u \\
\quad=2^{-1}[\alpha(x+0)+\alpha(x-0)]-2^{-1}[\alpha(0+)+\alpha(0-)]
\end{aligned}
$$

for all $x$ and 


$$
C \lim _{t \rightarrow 1} e^{|t C H|} f_{2}(x)=\phi(x) \text { for almost all } x \text {, respectively. }
$$

Combining the results of the two previous lemmas, we have

LemMa 3.4. According as $f(x)$ is in the form (1.1) or (1.2) we have

$$
\begin{aligned}
& \lim _{t \rightarrow 1} \int_{0}^{x} G e^{|\imath C B|} h(u) d u \\
& =2^{-1}[\alpha(x+0)+\alpha(x-0)]-2^{-1}[\alpha(0+)+\alpha(0-)-A G K x,
\end{aligned}
$$

$$
\lim _{t \rightarrow 1} G e^{|t C H|} h(x)=\phi(x)-A K G .
$$

We now determine $K$ in

Lemma 3.5.

$$
K=-2\left(A^{2} G \log 4\right)^{-1} \lim _{N \rightarrow \infty} \int_{N}^{2 N} \frac{-A t+B}{C^{2}+t^{2}} d \alpha^{t}(t)
$$

where $\alpha^{f}(x)$ is the right-hand side of (3.2).

The convergence of the integral (1.1) for $x=0$ implies

$$
\lim _{N \rightarrow \infty} \int_{N}^{2 N} \frac{-A t+B}{C^{2}+t^{2}} d\left[\alpha^{*}(t)+A K G t\right]=0 ;
$$

hence

$$
\begin{aligned}
\lim _{N \rightarrow \infty} \int_{N}^{2 N} \frac{-A t+B}{C^{2}+t^{2}} d \alpha^{t}(t) & =-\lim _{N \rightarrow \infty} A K G \int_{N}^{2 N} \frac{-A t+B}{C^{2}+t^{2}} d t \\
& =\frac{A^{2} K G}{2} \log 4 .
\end{aligned}
$$

This completes the proof of

THEOREM 3.1. (1) and (2) are inverted by (III) and (IV) respectively.

4. A complex inversion formula. We now indicate how the complex inversion formula (V) obtained heuristically may be established rigorously. We observe that we may write

$$
h(x)=(G 2 \pi)^{-1} \int \phi(y) d y \int e^{i u(x-y)} e^{-|C u|} d u-A K .
$$

After some calculation the left-hand side of (V) may be put in the form 


$$
\lim _{t \rightarrow 1} \pi^{-1} \int \frac{1-t}{(1-t)^{2}+(x-y)^{2}} \phi(y) d y-A K,
$$

but the integral above is known to tend to $\phi(x)$ for almost every $x$ $[1$, p. 550]. If $A$ is zero, $K$ is undefined but one may show that in this case the left-hand side of $(\mathrm{V})$ tends to $\phi(x)$ itself. These remarks establish

TheOREM 4.1. For almost all $x(2)$ is inverted by (V).

\section{BIBLIOGRAPHY}

1. H. Pollard, The Poisson transform, Trans. Amer. Math. Soc. vol. 78 (1955) pp. 541-550.

2. - Integral transforms, Duke Math. J. vol. 13 (1946) pp. 312 and 320.

UNION COLLEgE 